\title{
Germanica
}

\section{Autour de Michael Kohlhaas : réception et modernité de la nouvelle de Kleist dans l'Allemagne partagée de la fin des années soixante- dix}

Rezeption und Modernität der Kleist-Novelle « Michael Kohlhaas " im geteilten Deutschland der siebziger Jahre : Elisabeth Plessen und Christoph Hein.

Hélène Yèche

\section{OpenEdition}

Journals

Édition électronique

URL : http://journals.openedition.org/germanica/1817

DOI : 10.4000/germanica. 1817

ISSN : 2107-0784

\section{Éditeur}

Université de Lille

\section{Édition imprimée}

Date de publication : 30 juin 2004

Pagination : 145-154

ISBN : 9782913857131

ISSN : 0984-2632

Référence électronique

Hélène Yèche, « Autour de Michael Kohlhaas : réception et modernité de la nouvelle de Kleist dans l'Allemagne partagée de la fin des années soixante-dix », Germanica [En ligne], 34 | 2004, mis en ligne le 11 octobre 2012, consulté le 06 octobre 2020. URL : http://journals.openedition.org/germanica/1817 ; DOI : https://doi.org/10.4000/germanica.1817

Ce document a été généré automatiquement le 6 octobre 2020.

(c) Tous droits réservés 


\section{Autour de Michael Kohlhaas : réception et modernité de la nouvelle de Kleist dans l'Allemagne partagée de la fin des années soixante-dix ${ }^{1}$}

Rezeption und Modernität der Kleist-Novelle " Michael Kohlhaas » im geteilten Deutschland der siebziger Jahre : Elisabeth Plessen und Christoph Hein.

\section{Hélène Yèche}

1 Hasard de publication ou hommage implicite rendu à l'un des premiers écrivains modernes allemands à l'occasion du bicentenaire de sa naissance, la littérature allemande de la fin des années soixante-dix, à l'Ouest comme à l'Est, renoue avec le romantisme à travers le destin tourmenté et exemplaire de Michael Kohlhaas, héros de la célèbre nouvelle de Kleist: tandis que Elisabeth Plessen consacre au personnage historique un long roman sous le titre éponyme Kohlhaas en 1979, Christoph Hein publie un an plus tard à Berlin, dans le cadre de l'ancienne RDA, un petit récit, directement puisé aux sources de la littérature allemande classique, intitulé Le nouveau (mais plus heureux) Kohlhaas ${ }^{2}$. Un semblable choix poétique ne saurait laisser indifférent, accompli de conserve de part et d'autre de l'Elbe à une époque où précisément semble s'imposer l'émergence de deux littératures distinctes.

2 Le phénomène est d'abord intéressant en ce qu'il concerne deux jeunes talents littéraires : Elisabeth Plessen vient de publier un premier roman autobiographique très remarqué Mitteilung an den Adel (1976), tandis que Christoph Hein, connu jusque là principalement en tant que dramaturge, reçoit - pour une oeuvre en prose encore bien mince mais qui contient déjà en germes les lignes de force de son œuvre romanesque à venir -, le célèbre prix Heinrich-Mann. Issus d'une même génération, la génération symbole de l'Allemagne duelle née au sortir de la Seconde Guerre mondiale (tous deux sont nés en 1944), Elisabeth Plessen et Christoph Hein ont en fait grandi dans deux 
systèmes antagonistes, selon deux modèles idéologiques opposés, incarnant le partage contre-nature d'une nation. Pourtant leurs chemins semblent se croiser spontanément à la fin des années soixante-dix. En cette période troublée de l'histoire de la RFA, et probablement en réaction aux événements du tristement célèbre Deutscher Herbst (Automne allemand), leur intuition littéraire les conduit à mettre en scène, simultanément et sans concertation aucune, l'une des figures les plus fascinantes de l'histoire et de la littérature européenne : Kohlhaas, archétype de l'individu en révolte contre l'ordre établi, immortalisé par Heinrich von Kleist dans la nouvelle du même nom ${ }^{3}$.

3 Les deux textes procèdent à l'évidence d'une même source d'inspiration, s'articulant autour d'un même héros emprunté à la grande tradition littéraire allemande. Un examen attentif du titre de chacun des deux ouvrages permet cependant de cerner plus avant la perspective particulière adoptée par chaque auteur dans son entreprise de confrontation à l'héritage culturel allemand.

4 Au-delà du modèle littéraire, Elisabeth Plessen choisit de retourner sur les traces du personnage historique, ce Hans Kohlhase condamné en 1540 auquel on ne s'intéressa vraiment que cinquante ans après la mort de Kleist. Pourtant, en refusant un prénom à son personnage, l'auteur joue sur la double paternité, à la fois littéraire et historique. En effet, le titre choisi pour son roman ne renonce pas complètement à la référence littéraire puisque, malgré l'omission du prénom, l'orthographe du nom est reprise de la nouvelle de Kleist : Kohlhaas. Or un tel choix ne surprend guère. Il prouve simplement que le changement de genre affiché par l'écrivain pour son deuxième roman n'est que façade - du roman autobiographique, on passe en apparence au roman historique, mais en apparence seulement. Car c'est avant tout l'homme, l'individu qui intéresse E. Plessen. Fondé sur un patient travail d'archives, son roman est une reconstitution minutieuse du quotidien de l'individu dans l'Allemagne tourmentée du début du XvI siècle, l'Allemagne de Luther, de Melanchthon et de la Guerre des Paysans. L'auteur tente de recréer une atmosphère en mettant en scène l'enfance du personnage, en essayant d'imaginer au plus près de la réalité historique son entourage, les paysages qu'il a traversés et habités, en s'efforçant de reconstituer la vie d'une époque en somme, d'imaginer et de raconter sa propre version de l'histoire tragique d'un individu : «Est-ce que j'impose l'histoire ? Non. Je reconstruis, j'interviens $»^{4}$, explique le narrateur. Mais cette reconstruction, aussi fidèle qu'elle se veuille, laisse volontairement une grande part à la subjectivité. Le narrateur, qui se déclare en même temps auteur, l'avoue dès les premières lignes du roman: "J'écris cette histoire ainsi que je l'ai vue ${ }^{5}$. En réalité, le " je » domine, s'impose dès le départ, un " je » narratif mais réflexif aussi, qui tente de raconter comment on raconte, et qui cherche aussi par ce biais à définir ses propres contours, comme en témoigne ce passage : «Lui ou moi. Kohlhaas ou moi. Je réfléchis. Moi $»^{6}$. Plus que le modèle littéraire, c'est donc le personnage historique, et plus simplement encore l'individu qui fascine l'écrivain.

5 Christoph Hein en revanche donne à son récit un titre codé qui, une fois décrypté, fait office de dédicace et définit d'emblée sa position. Ce titre fait en effet doublement référence à Kleist. Il y est bien sûr ouvertement question du personnage central de la nouvelle de Kleist, ce Kohlhaas dont l'histoire est systématiquement transposé à l'époque contemporaine. Mais l'absence de prénom n'est pas liée cette fois au degré de réalité plus ou moins grand du personnage, posé ici comme un modèle, comme l'exemple type du révolté (d'ailleurs le véritable protagoniste du récit de Hein se 
prénomme Hubert). Elle s'explique par rapport à l'œuvre même de Kleist. Car Le nouveau (mais plus heureux) Kohlhaas évoque indéniablement un autre texte de Kleist, une anecdote pleine d'ironie intitulée Der neuere (glücklichere) Werther, publiée en 1881 dans les Berliner Abendblätter et écrite en référence à Goethe, au génie de la littérature allemande que Kleist rêvait de supplanter. La similitude des titres est trop frappante pour être pure coïncidence. Ce jeu intellectuel révèle à maints égards les intentions de l'auteur. En opérant cette subtile mise en abyme littéraire, Hein, écrivain contemporain de l'ancienne RDA, se place de facto, mais implicitement - à l'usage du lecteur averti qui saura décrypter l'allusion, et sans doute également à l'usage du censeur chargé de faire respecter les directives de la politique culturelle socialiste -, dans la lignée de deux des plus grands auteurs de langue allemande : le romantique Kleist, longtemps ignoré des artisans du réalisme socialiste, mais surtout, à travers lui, le grand Goethe, chef de file revendiqué de l'humanisme littéraire dont se réclame l'héritage culturel socialiste.

6 Si Hein n'hésite pas à afficher une filiation flatteuse, Elisabeth Plessen, plus modestement, contourne la référence littéraire pour s'attacher au personnage historique et à une approche originale de sa dimension humaine. Le positionnement respectif de l'auteur par rapport au modèle littéraire semble en fait révélateur du stade de développement de la littérature dans laquelle son œuvre s'inscrit: tandis que la jeune littérature socialiste, encore à la recherche d'une certaine légitimité, s'efforce de trouver un ancrage dans la grande tradition classique allemande en renouant avec des modèles plus ou moins agréés, la littérature ouest-allemande de la fin des années soixante-dix, après la politisation des années soixante et les déceptions qui ont suivi, voit conjointement le début d'une grave crise du roman et l'avènement d'une nouvelle subjectivité, d'un retour sur soi dont Elisabeth Plessen incarne le regard féminin, sans être pour autant féministe.

7 Dans un cas comme dans l'autre cependant, deux facteurs déterminants semblent avoir motivé la reprise d'une thématique traditionnelle et sa réécriture au présent de nos sociétés contemporaines. L'ancrage de l'histoire de référence dans un passé lointain et la personnalité marquante et exemplaire du héros se révèlent comme les deux véritables pôles de la modernité de Kleist en cette seconde moitié du xxe siècle. Même si les deux textes sont de nature très différente (un roman de 300 pages contre un récit de 15 pages), ils procèdent clairement d'un semblable retour sur le passé ayant pour point de départ le personnage tantôt historique, tantôt littéraire de Kohlhaas. Le choix poétique du retour en arrière, de la transposition, ce jeu avec différentes plages temporelles, le jeu de miroir entre passé et présent qui en résulte est à l'évidence le principal dénominateur commun aux deux textes. On perçoit dans un cas comme dans l'autre la volonté d'observer et de réfléchir le présent à travers le prisme à la fois protecteur et révélateur du passé. Car c'est bien du présent qu'il est toujours question malgré le travestissement historique, toute littérature n'étant en fin de compte - mais c'est aussi en cela que réside l'essentiel de sa fonction - que le reflet de la société dont elle procède.

8 Sous le masque du roman historique, Elisabeth Plessen réagit à sa manière aux débordements de l'action terroriste qui ont bouleversé la République Fédérale durant les années soixante-dix et plus particulièrement à l'automne 1977, tandis que Hein, étranger par la force des choses à ce phénomène terrible qui frappe l'autre moitié de l'Allemagne, recourt, probablement non sans arrière-pensée, à la même trame pour 
fustiger, sous des dehors en apparence tout à fait neutres, les structures par trop dirigistes de l'organisation socialiste.

Ce n'est d'ailleurs pas un hasard si le récit de Ch. Hein, publié en 1980, se situe chronologiquement au début de la décennie précédente et coïncide donc précisément avec les premières années du terrorisme de la Bande à Baader, à savoir 1972/73. La relation au temps présent est signalée d'emblée, dès le sous-titre : Rapport sur une affaire judiciaire des années 1972/737. L'action se situe donc dans l'ancienne République démocratique allemande au début des années soixante-dix où l'auteur transpose l'histoire de Kohlhaas, cet homme à la fois profondément sincère et droit, mais également d'une constance terrifiante dans sa quête aveugle de justice qui aboutira à son auto-destruction. Hein conserve volontairement la trame de la nouvelle de Kleist dans ses grandes lignes. Son nouveau Kohlhaas occupe un petit emploi de comptable dans une VEB. En raison d'une absence pour maladie, il voit sa prime annuelle diminuée de $40 \mathrm{DM}$. S'estimant lésé par le système, il intente plusieurs procès à l'entreprise, d'abord sans succès, ne parvenant qu'à faire le vide autour de lui et à briser son ménage, pour obtenir finalement gain de cause grâce à une curieuse intervention extérieure. On retrouve aisément les principaux éléments du récit premier dans lequel un homme, victime d'une injustice relative, entame un procès qui n'aboutit pas et décide alors de faire lui-même justice, basculant irrémédiablement dans l'illégalité.

Certes le Kohlhaas de Hein n'a pas l'envergure de son modèle : il ne met pas le pays à feu et à sang et présente une personnalité fade et banale, très loin de la dualité mystérieuse du Michael Kohlhaas de Kleist annoncée dans l'incipit : « Sur les rives de la Havel vivait, au milieu du $\mathrm{xVI}^{\mathrm{e}}$ siècle, un marchand de chevaux du nom de Michael Kohlhaas, fils d'un maître d'école, un des êtres les plus honnêtes et en même temps les plus effrayants de son temps ${ }^{8}$.» Le Kohlhaas de Hein se présente plutôt comme une pâle copie de l'original : « Le décor se situe à H., une ville de la forêt de Thuringe, au bord de la haute Werra, arrondissement du district de Suhl. Hubert K., qui était comptable à la fabrique de chaises nationalisée de la ville, marié et père d'un petit garçon, était considéré par ses voisins et collègues comme un modèle du genre ${ }^{9}$. "

11 Le conflit évolue d'abord selon un schéma identique : comme à la suite de la rencontre de Kohlhaas avec Luther, Hubert K. songe un instant à retirer sa plainte, mais il est déjà allé trop loin au regard du monde, et c'est sous la pression muette du jugement des autres que s'exacerbe le conflit de l'individu avec l'autorité de l'État. C'est la fin du récit qui diverge fondamentalement par rapport au texte de Kleist: l'intervention quasi magique d'un deus ex machina en la personne de Johannes B. produit un renversement de l'histoire tout à fait surprenant qui conduit à la révision inattendue du procès et va en apparence à l'encontre de la thèse de la nouvelle de Kleist qui scelle l'impossibilité de revenir en ce monde sur une décision de justice et laisse Kohlhaas prouver par la mort son bon droit. Ici, il semblerait au contraire que la société socialiste ait trouvé le moyen de garantir à ses citoyens un soutien incontesté, surtout lorsque ceux-ci semblent désespérer d'elle : «Je n'ai plus aucune confiance dans la légalité socialiste », avait déclaré Hubert $\mathrm{K}^{10}$.

Mais le ton général du récit est trop ironique pour ne pas lire cette chute comme destinée à provoquer la réaction du lecteur et à déclencher en lui l'amorce d'une réflexion. C'est d'ailleurs un procédé que Hein affectionne particulièrement et que l'on retrouve dans l'épilogue de son roman Le Joueur de tango, comme dans tout le discours à double entente de l'héroïne de son premier grand succès L'ami étranger ${ }^{11}$. Le happy end, 
qui rappelle l'optimisme de rigueur dans la politique culturelle des années soixante prônant les héros positifs du réalisme socialiste, n'est chez Hein qu'une façade qui cache mal l'abîme de solitude du personnage et sa victoire à la Pyrrhus.

Hein conduit en effet son récit avec une distance ironique manifeste. Le style sobre, soutenu et volontairement archaïsant, contraste avec la banalité du motif. Mais plus significative encore est l'attitude volontairement neutre du narrateur qui s'abstient de tout jugement, ce style administrativement sec et dépouillé, celui-là même des procèsverbaux, qui se contentent de rendre compte d'une série d'événements sur un ton neutre et impartial, sine ira et studio, sans haine ni passion, à l'image de ces chroniqueurs d'antan dont Hein aime à se réclamer. L'auteur ménage volontairement une distance entre le narrateur, qui se pose comme un observateur neutre, et le héros, décrit comme objet de son rapport. Le narrateur utilise volontiers le nous de majesté ${ }^{12}$. De cette distance naît une ironie qui convie plus nettement à la relecture qu'à l'identification.

14 Le titre du récit, qui contient déjà une référence non négligeable à l'histoire littéraire allemande, se révèle lui aussi après-coup doublement ironique : car le bonheur du nouveau Kohlhaas, d'ailleurs mis entre parenthèses dès le titre, ce qui est un signe, apparaît finalement comme bien ténu et tout relatif. Or il n'est pas seulement imputable à la réécriture dans un sens positif/mélioratif d'un texte connu, à l'instar de Kleist dont le nouveau Werther présente la problématique de l'impossible relation à trois revue et corrigée : la fin est idyllique, le barbon meurt à l'occasion de la tentative de suicide manquée du jeune rival et les amoureux convolent en justes noces... La fin, heureuse en apparence, du récit de Hein n'obéit pas non plus aux impératifs du réalisme socialiste dont l'écrivain, sous des dehors très sages, faisait déjà fi à l'époque car son héros, divorcé, est réduit à la fin du récit par la bouche de la grand-mère de son ex-femme à un verfluchter Gottesnarr (un sacré fou de Dieu) et ainsi irrémédiablement condamné par le bon sens populaire qu'incarne cette dernière.

Le bonheur du nouveau Kohlhaas est tout simplement mis en doute par l'auteur. Au fur et à mesure qu'avance le récit se dessine en filigrane une critique sociale masquée jusque là par le caractère positif du titre mais que corrobore sans conteste le choix premier de l'adaptation de ce récit dramatique de Kleist. Le recours à des sujets historiques puisés dans le fonds culturel européen était un procédé classique dans la littérature de l'ancienne RDA, au théâtre en particulier, dans le but pragmatique de contourner la censure. En même temps, par ce retour au romantisme allemand, Hein prend position en faveur d'un élargissement de l'héritage culturel au-delà du classicisme de Weimar, vers les sphères plus diffuses du romantisme, dans un élan de redécouverte initié par Ch. Wolf (Kein Ort. Nirgends) et poursuivi à la même époque par U. Plenzdorf (Die neuen Leiden des jungen W.). C'est en ce sens que, dans son cas, la double référence à Goethe et à Kleist demeure très ambiguë. Car en choisissant de raconter l'histoire du héros tragique de Kleist, revue et corrigée de manière positive à la lumière de la société socialiste progressiste, Hein s'assurait a priori un certain crédit dû à la renommée de l'auteur de référence tout en prévenant la méfiance des autorités par l'affirmation du caractère positif de l'événement transposé, comme pour excuser la référence interdite.

16 Cependant la distance ironique vis-à-vis du personnage et la neutralité affichée du compte rendu ne font que renforcer l'impression de malaise qui envahit le lecteur devant tant de bonne volonté de la part des autorités et tant d'abnégation de la part du 
héros : comment se satisfaire d'une victoire qui a exigé tant de sacrifices ? Le succès du nouveau Kohlhaas remet indirectement en question le fonctionnement pseudodémocratique de la société socialiste tout en posant le problème du degré d'engagement social réel de tout citoyen. Mais comme Hein n'est pas un dissident, il évite de tomber dans le piège d'une dénonciation simpliste, et sa critique peut aussi, paradoxalement, au-delà de l'ironie manifeste du propos, être interprétée dans un sens constructif. Hein renvoie finalement dos à dos citoyen et société, dénonçant le danger de l'individualisme aveugle sans épargner la tutelle administrative socialiste.

Et c'est d'ailleurs dans la mise en évidence de comportements extrémistes que les deux textes étudiés se rejoignent de la manière la plus évidente, devenant textes historiques d'actualité. La fascination pour la rébellion est tempérée par les ravages que peut provoquer l'obstination bornée d'un seul individu capable de mettre en péril un système social tout entier. C'est parce que l'histoire semble une fois encore se répéter que Hein et Plessen s'intéressent dans les années soixante-dix à la personnalité d'un individu en révolte contre un système social qu'il estime hostile. L'entreprise des deux écrivains a pour corollaire une volonté critique qui s'exerce plus à l'encontre de l'individu que d'un modèle social. Il s'agit moins de condamner un système, qu'il soit socialiste ou capitaliste, que de remonter à la source même du malaise : la position, le point de vue de l'individu. Il en résulte une mise en perspective de la violence gratuite contemporaine à travers le prisme de la distance historique. Le retour en arrière, métaphorique ou volontairement historicisé, permet de convoquer le passé pour mieux comprendre le présent.

18 Et pour ce faire chaque auteur use de sa propre stratégie narrative. Le roman d'E. Plessen est d'accès difficile car touffu. Sa structure est inspirée des romans classiques comme en témoigne la division en chapitres avec titres synoptiques. Il est écrit dans une langue volontiers archaïsante, mais qui joue aussi sur les anachronismes, comme pour bien nous rappeler que son auteur parle du présent qui est le nôtre ${ }^{13}$. Et de fait, l'histoire de Kohlhaas devient un peu la nôtre, grâce à une écriture vecteur d'identification qui convoque systématiquement la subjectivité, celle de l'auteur comme celle du lecteur. E. Plessen s'immisce dans l'être le plus intime de son personnage, tentant d'inventer ou de retrouver ses rêves, se permettant plusieurs variantes de l'histoire, des corrections opportunes que seule l'imagination d'un écrivain peut se permettre ${ }^{14}$. Il y a donc chez E. Plessen une volonté pédagogique affirmée qui se situe aux antipodes du roman historique traditionnel.

Chez Hein, le récit se propose à plusieurs niveaux de lecture. Malgré un titre savant et rassurant, la chute demeure surprenante et provocatrice. Elle doit inciter le lecteur à une relecture critique de l'ensemble. Or cette trame correspond très exactement au principe dialogique sur lequel repose l'essentiel de la poétique de Hein ${ }^{15}$ : engager le dialogue avec le lecteur, l'inciter à entamer une réflexion autonome par le biais d'une provocation. D'ailleurs, dans le cas précis de ce petit récit, la provocation ne s'adresse pas qu'au lecteur, elle vaut aussi semble-t-il pour d'autres instances. Hein s'inscrit volontairement dans une double tradition littéraire allemande, convoquant à la fois Kleist et Goethe. Or le fait même de revendiquer cette double paternité peut être interprété comme un pied de nez à la tradition littéraire socialiste. La démarche de Ch. Hein mise en évidence ici s'inscrit en fait dans la mouvance de la littérature contestataire de l'époque (U. Plenzdorf, Ch. Wolf, G. de Bruyn, I. Morgner...) qui, à l'Est, a tenté d'initier un nouveau mode de réception de l'héritage classique pour dénoncer la 
corruption des idéaux humanistes de l'époque classique dans tous les domaines de la réalité socialiste et prôner la recherche d'un socialisme "éclairé » avant la révolution de l'automne 1989.

Le mouvement terroriste qui a ensanglanté l'Allemagne au milieu des années 70 a naturellement constitué un thème de prédilection pour la littérature allemande à partir de cette période. La fascination qui s'est exercée sur nombre d'écrivains (Böll, Fried, Andersch...) ne se dément pas jusqu'à aujourd'hui, puisque même après la réunification, alors que d'autres foyers brûlants préoccupent les esprits (chômage, exclusion, montée de l'extrême droite), un auteur comme F.C. Delius termine une trilogie sur l'Automne allemand en publiant le troisième volet intitulé Himmelfahrt eines Staatsfeindes (1992).

Dans le cas présent cependant, la confrontation avec la violence extrême telle qu'elle a été pratiquée par la bande à Baader se mue, à travers la transposition littéraire, en une volonté de critique individuelle et sociale qui dépasse la simple condamnation stérile. Tandis que le roman historique d'Elisabeth Plessen tente de retrouver l'authenticité subjective du fauteur de trouble tout en forçant l'identification de manière saisissante, le récit de $\mathrm{Ch}$. Hein tourne en ridicule la fascination pour la rébellion, tout en plaidant pour un autre engagement social (ou socialiste?) de l'individu.

La réception de Kleist dans l'Allemagne partagée de la seconde moitié $\mathrm{du} \mathrm{xx}^{\mathrm{e}}$ siècle prouve en tout cas que les littératures ne sont pas étrangères l'une à l'autre, ni dans le temps, ni dans l'espace, mais s'interpénètrent et s'enrichissent en un savant jeu de miroirs. Ainsi, c'est dans le recours et le retour au romantisme, à une certaine forme de sécession incarnée par Heinrich von Kleist, que se rejoignent à la fin des années soixante-dix les littératures allemandes face à la menace terroriste. Dans ce rapprochement entre passé et présent, mais surtout entre littérature de l'Est et littérature de l'Ouest, réside probablement la plus grande modernité de Kleist.

\section{NOTES}

1. Cet article est la version française légèrement modifiée de la contribution suivante : H. Yèche : «Rezeption und Modernität der Kleist-Novelle « Michael Kohlhaas» im geteilten Deutschland der siebziger Jahre : Elisabeth Plessen und Christoph Hein », in : Ensberg, Peter und Marquardt, Hans-Jochen (Editors) : Kleists Beitrag zur Ästhetik der Moderne. III. Frankfurter Kleist-Kolloquium, 16-17.10.1998, Kleist-Gedenk- und Forschungsstätte [Kleist-Museum], Stuttgart, Akademischer Verlag Hans-Dieter Heinz, 2002, S. 73-82.

2. Toutes les références citées sont tirées des éditions suivantes: Elisabeth Plessen : Kohlhaas, Roman, Fischer, Frankfurt am Main, 1987; Christoph Hein: «Der neuere (glücklichere) Kohlhaas », in : Nachtfahrt und früher Morgen, Prosa, dtv, München, 1987, S.102-117.

3. Heinrich von Kleist: Michael Kohlhaas. Aus einer alten Chronik (1808-1810), Novelle, Reclam, Ditzingen.

4. Cf. E. Plessen, Op. cit., S. 54 : «Schreibe ich Geschichte vor? Nein. Ich rekonstruiere, ich greife ein. "

5. Cf. E. Plessen, Op. cit., S. 9 : «Ich schreibe diese Geschichte, ich habe sie so gesehen. »

6. Cf. E. Plessen, Op. cit., S. 24 : «Er oder ich. Kohlhaas oder ich. Ich überlege es mir. Ich.» 
7. Cf. Ch. Hein, Op. cit., S. 102 : Bericht über einen Rechtshandel aus den Jahren 1972/73.

8. Cf. Heinrich von Kleist, Op. cit. : «An den Ufern der Havel lebte, um die Mitte des sechzehnten Jahrhunderts, ein Roßhändler, namens Michael Kohlhaas, Sohn eines Schulmeisters, einer der rechtschaffensten zugleich und entsetzlichsten Menschen seiner Zeit. »

9. Cf. Ch. Hein, Op. cit., S. 102 : «Die Szene ist H., eine Stadt im Thüringischen, gelegen an der oberen Werra, Kreisstadt des Verwaltungsbezirks Suhl. Hubert K., Buchhalter in der volkseigenen Stuhlfabrik der Stadt, verheiratet und Vater eines Sohnes, galt bei seinen Nachbarn und Kollegen als das Muster eines Rechnungsführers. "

10. Cf. Ch. Hein, Op. Cit., S. 115: «Ich habe kein Vertrauen mehr in die sozialistische Gesetzlichkeit. »

11. Christoph Hein : Der Tangospieler, Roman, Aufbau-Verlag, Berlin und Weimar, 1989 ; Christoph Hein : Der fremde Freund, Novelle, Aufbau-Verlag, Berlin und Weimar, 1982.

12. Cf. Ch. Hein, Op. Cit, S. 104, 111, 112 : « wenn wir von gelegentlichen Anmerkungen Hubert K.s absehen/diese Vermutung in unseren Bericht mit aufzunehmen/weitaus schwieriger gestaltete sich für unseren Helden das gemeinsame Leben mit Elvira ».

13. Des anachronismes patents (count down, langer Marsch, Wolkenkratzer, fliegende Untertassen, Marsmenschen...) voisinent avec des expressions oubliées (Sie der Heerholt der dieberey, fantasey, so thu ich nachher kriechen...). Cf. E. Plessen, Op. cit, S. 233, 140, 112, 260 ..

14. Cf. E. Plessen, Op. cit, S. 53 : «Nein, anders. Gegenentwurf.» « Non, autrement. Contreprojet. "

15. Cf. Hélène Guibert-Yèche : Christoph Hein, l'œuvre romanesque des années 1980. De la provocation au dialogue, Etudes et Documents 39, Coll. Contacts (dir. Jean-Marie Valentin), Ed. Peter Lang, 1998, 402 p.

\section{RÉSUMÉS}

La réception de la célèbre nouvelle de Kleist Michael Kohlhaas (1808-1810) dans l'Allemagne partagée de la fin des années soixante-dix, à travers la publication du roman historique de $\mathrm{E}$. Plessen Kohlhaas (1979) et du bref récit en prose de Ch. Hein Der neuere (glücklichere) Kohlhaas (1980), montre bien la force et le rayonnement de ce texte classique dans nos sociétés modernes. La réapparition de l'archétype de l'individu révolté sur la scène littéraire allemande de l'époque n'est pas étrangère au phénomène terroriste qui a secoué durablement la scène politique et sociale en Allemagne de l'Ouest, même si les répercussions ont été moindres à l'Est. Mis en scène avec des moyens et selon des principes poétiques différents mais à partir d'un constat commun les dangers de l'individualisme terroriste -, le retour en force du Kohlhaas de Kleist des deux côtés du Mur de Berlin marque peut-être le rapprochement de deux littératures trop vite cataloguées comme divergentes.

Die Rezeption der berühmten Kleist-Novelle Michael Kohlhaas (1808-1810) im geteilten Deutschland am Ende der siebziger Jahre mit der gleichzeitigen Veröffentlichung des historischen Romans von E. Plessen Kohlhaas (1979) und der Erzählung von Ch. Hein Der neuere (glücklichere) Kohlhaas (1980) ist ein deutlicher Beweis für die Tragweite dieses Klassikers in unseren modernen Gesellschaften. Das Wiederaufleben des Archetyps eines Aufrührers in der damaligen deutschsprachigen Literatur lässt sich wohl durch die Terrorangriffe erklären, die West-Deutschland politisch und sozial erschüttert haben, auch wenn Ost-Deutschland weniger 
betroffen wurde. Die Wiederaufnahme von Kleists Kohlhaas in beiden deutschen Staaten mit unterschiedlichen poetischen Mitteln, aber mit demselben Ausgangspunkt - der Gefahr des Terrorindividualismus -, bedeutet vielleicht, dass die beiden Literaturen einander nicht so fremd waren.

\section{AUTEUR \\ HÉLÈNE YÈCHE}

Université de Poitiers 